\title{
Some formulas of variance of uncertain random variable
}

\author{
Yuhong Sheng ${ }^{1 *}$ and Kai Yao ${ }^{2}$
}

\author{
${ }^{*}$ Correspondence: \\ sheng-yh12@mails.tsinghua.edu.cn \\ 1 Department of Mathematical \\ Sciences, Tsinghua University, \\ Beijing 100084, China \\ Full list of author information is \\ available at the end of the article
}

\begin{abstract}
Uncertainty and randomness are two basic types of indeterminacy. Chance theory was founded for modeling complex systems with not only uncertainty but also randomness. As a mixture of randomness and uncertainty, an uncertain random variable is a measurable function on the chance space. It is usually used to deal with measurable functions of uncertain variables and random variables. There are some important characteristics about uncertain random variables. The expected value is the average value of uncertain random variable in the sense of chance measure and represents the size of uncertain random variable. The variance of uncertain random variable provides a degree of the spread of the distribution around its expected value. In order to describe the variance of uncertain random variable, this paper provides some formulas to calculate the variance of uncertain random variables through chance distribution and inverse chance distribution. Several practical examples are also provided to calculate the variance for through chance distribution inverse chance distribution.
\end{abstract}

Keywords: Chance theory; Uncertain random variable; Inverse chance distribution; Variance

\section{Introduction}

Probability theory is a branch of mathematics for studying the behavior of random phenomena. In order to deal with randomness, Kolmogorov [1] defined a probability measure as a set function satisfying the following three axioms: (i) normality, (ii) nonnegativity, and (iii) additivity. Before applying it in practice, we should first obtain the probability distribution via statistics or test the probability distribution to make sure it is close enough to the real frequency, either of which is based on a lot of observed data. However, due to the technological or economical difficulties, we sometimes have no observed data. In this case, we have to rely on domain experts evaluating their belief degree about the chances that the possible events happen. According to Kahneman and Tversky [2], human tends to overweight unlikely events, so the belief degree generally has a much larger range than the real frequency. As a result, the probability theory is not applicable in this case; otherwise, it may lead to counterintuitive results [3]. Liu [4] gave some examples of it.

The uncertainty theory is a branch of mathematics for modeling belief degrees. In order to deal with human uncertainty, Liu [5] first presented uncertain measure as a set function satisfying four measure axioms: (i) normality, (ii) duality, (iii) subadditivity, and (iv) product axiom [6]. As a fundamental concept in uncertainty theory, the uncertain variable

(c) 2014 Sheng and Yao; licensee Springer. This is an Open Access article distributed under the terms of the Creative Commons Attribution License (http://creativecommons.org/licenses/by/4.0), which permits unrestricted use, distribution, and reproduction in any medium, provided the original work is properly credited. 
was presented by Liu [5] in 2007. In order to describe the uncertain variable, Liu [5] introduced the concept of uncertainty distribution. Liu [3] proposed the concept of inverse uncertainty distribution, and Liu [4] verified a sufficient and necessary condition for it. After that, many researchers widely studied the uncertainty theory and made significative progress. Gao [7] studied the properties of continuous uncertain measure. Peng and Iwamura [8] proved a sufficient and necessary condition for uncertainty distribution. Furthermore, a measure inversion theorem was given by Liu [3] that may yield uncertain measures of some special events from the uncertainty distribution of the corresponding uncertain variable. In addition, the concept of independence was proposed by Liu [6]. After, Liu [3] presented the operational law of uncertain variables. In order to rank uncertain variables, Liu [5] proposed the concept of expected value of uncertain variables. The linearity of expected value operator was verified by Liu [3]. As an important contribution, Liu and $\mathrm{Ha}$ [9] derived a useful formula for calculating the expected values of strictly monotone functions of independent uncertain variables. Meanwhile, Liu [5] presented the concept of variance of uncertain variables and also proposed some formulas to calculate the variance through uncertainty distribution. Recently, Yao [10] proposed a formula to calculate the variance using inverse uncertainty distribution. Sheng and Kar [11] verified some results of moment of uncertain variable through inverse uncertainty distribution.

However, in many cases, randomness and uncertainty exist simultaneously in a complex system. In order to describe such a system, Liu [12] pioneered the concepts of uncertain random variable and chance measure. Meanwhile, Liu [12] defined the concepts of chance distribution, expected value and variance for uncertain random variable. Following that, Liu [13] presented the operational law of uncertain random variable, and proposed uncertain random programming as a branch of mathematical programming involving uncertain random variables. In addition, Guo and Wang [14] proved a formula for calculating the variance of uncertain random variables. Yao and Gao [15] verified a law of large numbers for uncertain random variables of independent and identical distribution. Gao and Yao [16] proposed uncertain random process.

This paper further studies the variance of uncertain random variables and provides some formulas to calculate the variance through inverse chance distribution. The rest of this paper is organized as follows: Section 'Preliminary' presents some basic concepts and theorems about uncertain random variables and recalled some concepts of expected value, variance, and so on. Some formulas about the variance are derived through chance distribution and inverse chance distribution in Section 'Variance of uncertain random variable.' Finally, some conclusions are given in Section 'Conclusions'.

\section{Preliminary}

In this section, we will introduce some useful definitions and theorems about random variable, uncertain variable and uncertain random variable.

\section{Random variable}

Let $\mathcal{A}$ be a $\sigma$-algebra on a nonempty set $\Omega$. A set function $\operatorname{Pr}: \mathcal{A} \rightarrow[0,1]$ is called a probability measure if it satisfies the following axioms:

Axiom 1: (Normality axiom) $\operatorname{Pr}\{\Omega\}=1$ for the universal set $\Omega$.

Axiom 2: (Nonnegativity axiom) $\operatorname{Pr}\{\mathcal{A}\} \geq 0$ for any event $\mathcal{A}$. 
Axiom 3: (Additivity axiom) For every countable sequence of events $\mathcal{A}_{1}, \mathcal{A}_{2}, \cdots$, we have

$$
\operatorname{Pr}\left\{\bigcup_{i=1}^{\infty} \mathcal{A}_{i}\right\}=\sum_{i=1}^{\infty} \operatorname{Pr}\left\{\mathcal{A}_{i}\right\} .
$$

Definition 1. (Kolmogorov [1]) The set function Pr is called a probability measure if it satisfies the normality, nonnegativity, and additivity axioms.

Besides, the product probability on the product $\sigma$-algebra $\mathcal{A}$ is defined as follows:

Let $\left(\Omega_{k}, \mathcal{A}_{k}, \operatorname{Pr}_{k}\right)$ be probability spaces for $k=1,2, \ldots$. It has been proven that there is a unique probability measure Pr on the product $\sigma$-algebra $\mathcal{A}$ such that

$$
\operatorname{Pr}\left\{\prod_{i=1}^{\infty} \mathcal{A}_{k}\right\}=\prod_{k=1}^{\infty} \operatorname{Pr}\left\{\mathcal{A}_{k}\right\}
$$

where $\mathcal{A}_{k}$ are arbitrarily chosen events from $\mathcal{A}_{k}$ for $k=1,2, \cdots$, respectively. This conclusion is called product probability theorem. Such a probability measure is called product probability measure, denoted by

$$
\operatorname{Pr}=\underset{1}{\operatorname{Pr}} \times \underset{2}{\operatorname{Pr}} \times \cdots
$$

\section{Uncertain variable}

Definition 2. (Liu [5]) Let $\mathcal{L}$ be a $\sigma$-algebra on a nonempty set $\Gamma$. A set function $\mathcal{M}: \mathcal{L} \rightarrow$ $[0,1]$ is called an uncertain measure if it satisfies the following axioms:

Axiom 1: (Normality axiom) $\mathcal{M}\{\Gamma\}=1$ for the universal set $\Gamma$.

Axiom 2: (Duality axiom) $\mathcal{M}\{\Lambda\}+\mathcal{M}\left\{\Lambda^{c}\right\}=1$ for any event $\Lambda$.

Axiom 3: (Subadditivity axiom) For every countable sequence of events $\Lambda_{1}, \Lambda_{2}, \cdots$, we have

$$
\mathcal{M}\left\{\bigcup_{i=1}^{\infty} \Lambda_{i}\right\} \leq \sum_{i=1}^{\infty} \mathcal{M}\left\{\Lambda_{i}\right\} .
$$

Besides, the product uncertain measure on the product $\sigma$-algebra $\mathcal{L}$ is defined by Liu [6] as follows:

Axiom 4: (Product axiom) Let $\left(\Gamma_{k}, \mathcal{L}_{k}, \mathcal{M}_{k}\right)$ be uncertainty spaces for $k=1,2, \cdots$. Then the product uncertain measure $\mathcal{M}$ is an uncertain measure satisfying

$$
\mathcal{M}\left\{\prod_{i=1}^{\infty} \Lambda_{k}\right\}=\bigwedge_{k=1}^{\infty} \mathcal{M}_{k}\left\{\Lambda_{k}\right\}
$$

where $\Lambda_{k}$ are arbitrarily chosen events from $\mathcal{L}_{k}$ for $k=1,2, \cdots$, respectively.

An uncertain variable is essentially a measurable function from an uncertainty space to the set of real numbers. In order to describe an uncertain variable, a concept of uncertainty distribution is defined as follows.

Definition 3. (Liu [5]) Let $\xi$ be an uncertain variable. Then its uncertainty distribution is defined by

$$
\Phi(x)=\mathcal{M}\{\xi \leq x\}
$$

for any real number $x$. 
An uncertainty distribution $\Phi$ is said to be regular if its inverse function $\Phi^{-1}(\alpha)$ exists and is unique for each $\alpha \in(0,1)$. Inverse uncertainty distribution plays an important role in the operation of independent uncertain variables.

The expected value of uncertain variable is the average value of uncertain variable in the sense of uncertain measure and it represents the size of uncertain variable. The variance of uncertain variable provides a degree of the spread of the distribution around its expected value.

Definition 4. (Liu [5]) The expected value of an uncertain variable $\xi$ is defined by

$$
E[\xi]=\int_{0}^{+\infty} \mathcal{M}\{\xi \geq x\} \mathrm{d} x-\int_{-\infty}^{0} \mathcal{M}\{\xi \leq x\} \mathrm{d} x
$$

provided that at least one of the two integrals is finite.

Theorem 1. (Liu [5]) Let $\xi$ be an uncertain variable with uncertainty distribution $\Phi$. If the expected value exists, then

$$
E[\xi]=\int_{0}^{+\infty}(1-\Phi(x)) \mathrm{d} x-\int_{-\infty}^{0} \Phi(x) \mathrm{d} x .
$$

The expected value of the linear uncertain variable $\xi \sim \mathcal{L}(a, b)$ is $E[\xi]=(a+b) / 2$; the expected value of the normal uncertain variable $\xi \sim \mathcal{N}(e, \sigma)$ is $E[\xi]=e$; and the expected value of the lognormal uncertain variable $\xi \sim \mathcal{L} \mathcal{O} \mathcal{N}(e, \sigma)$ is

$$
E[\exp (\xi)]= \begin{cases}\sqrt{3} \sigma \exp (e) \csc (\sqrt{3} \sigma), & \text { if } \sigma \leq \pi / \sqrt{3} \\ +\infty, & \text { otherwise. }\end{cases}
$$

In 2010, Liu [3] first introduced a formula for expected value by inverse uncertainty distribution, that is

$$
E[\xi]=\int_{0}^{1} \Phi^{-1}(\alpha) \mathrm{d} \alpha .
$$

Liu and $\mathrm{Ha}$ [9] proposed a generalized formula for expected value by inverse uncertainty distribution.

Theorem 2. (Liu and Ha [9]) Let $\xi_{1}, \xi_{2}, \ldots, \xi_{n}$ be independent uncertain variables with uncertainty distributions $\Phi_{1}, \Phi_{2}, \cdots, \Phi_{n}$, respectively. If $f\left(\xi_{1}, \xi_{2}, \cdots, \xi_{n}\right)$ is strictly increasing with respect to $\xi_{1}, \xi_{2}, \cdots, \xi_{m}$ and strictly decreasing with respect to $\xi_{m+1}, \xi_{m+2}, \cdots, \xi_{n}$, then the uncertain variable $\xi=f\left(\xi_{1}, \xi_{2}, \cdots, \xi_{n}\right)$ has an expected value

$$
E[\xi]=\int_{0}^{1} f\left(\Phi_{1}^{-1}(\alpha), \cdots, \Phi_{m}^{-1}(\alpha), \Phi_{m+1}^{-1}(1-\alpha), \cdots, \Phi_{n}^{-1}(1-\alpha)\right) \mathrm{d} \alpha .
$$

Based on this result, Liu [6] proved the linearity property of the expected value operator. For two independent uncertain variables $\xi$ and $\eta$, we have $E[a \xi+b \eta]=a E[\xi]+b E[\eta]$ where $a$ and $b$ are real numbers.

Definition 5. (Liu [5]) Let $\xi$ be an uncertain variable with finite expected value $E[\xi]$. Then the variance of $\xi$ is

$$
V[\xi]=E\left[(\xi-E[\xi])^{2}\right] .
$$


Since the uncertain measure is a subadditivity measure, the variance of uncertain variable cannot be derived simply by the uncertainty distribution. In this case, Liu [5] suggested to accept the following formulas as a stipulation

$$
V[\xi]=\int_{0}^{+\infty}[1-\Phi(E[\xi]+\sqrt{x})+\Phi(E[\xi]-\sqrt{x})] \mathrm{d} x .
$$

Based on this stipulation, the variance of the linear uncertain variable $\xi \sim \mathcal{L}(a, b)$ is $V[\xi]=(b-a)^{2} / 12$ and the variance of the normal uncertain variable $\xi \sim \mathcal{N}(e, \sigma)$ is $V[\xi]=\sigma^{2}$.

In 2013, Yao [10] proved a formula to calculate the variance of an uncertain variable via the inverse uncertainty distribution.

Theorem 3. (Yao [10]) Let $\xi$ be an uncertain variable with a regular uncertainty distribution $\Phi$. If the expected value $E[\xi]$ exists, then the variance is

$$
V[\xi]=\int_{0}^{1}\left(\Phi^{-1}(\alpha)-E[\xi]\right)^{2} \mathrm{~d} \alpha .
$$

\section{Uncertain random variable}

In 2013, Liu [12] first proposed chance theory, which is a mathematical methodology for modeling complex systems with both uncertainty and randomness, including chance measure, uncertain random variable, chance distribution, operational law, expected value, and so on. The chance space refers to the product $(\Gamma, \mathcal{L}, \mathcal{M}) \times(\Omega, \mathcal{A}, \operatorname{Pr})$, in which $(\Gamma, \mathcal{L}, \mathcal{M})$ is an uncertainty space and $(\Omega, \mathcal{A}, \operatorname{Pr})$ is a probability space.

Definition 6. (Liu [12]) Let $(\Gamma, \mathcal{L}, \mathcal{M}) \times(\Omega, \mathcal{A}, \operatorname{Pr})$ be a chance space, and let $\Theta \in \mathcal{L} \times \mathcal{A}$ be an uncertain random event. Then the chance measure of $\Theta$ is defined as

$$
\operatorname{Ch}\{\Theta\}=\int_{0}^{1} \operatorname{Pr}\{\omega \in \Omega \mid \mathcal{M}\{\gamma \in \Gamma \mid(\gamma, \omega) \in \Theta\} \geq r\} \mathrm{d} r .
$$

Liu [12] proved that a chance measure satisfies normality, duality, and monotonicity properties, that is

(i) $\operatorname{Ch}\{\Gamma \times \Omega\}=1$;

(ii) $\operatorname{Ch}\{\Theta\}+\operatorname{Ch}\left\{\Theta^{c}\right\}=1$ for and event $\Theta$;

(iii) $\operatorname{Ch}\left\{\Theta_{1}\right\} \leq \operatorname{Ch}\left\{\Theta_{2}\right\}$ for any real number set $\Theta_{1} \subset \Theta_{2}$.

Besides, Hou [17] proved the subadditivity of chance measure, that is,

$$
\operatorname{Ch}\left\{\bigcup_{i=1}^{\infty} \Theta_{i}\right\} \leq \sum_{i=1}^{\infty} \operatorname{Ch}\left\{\Theta_{i}\right\}
$$

for a sequence of events $\Theta_{1}, \Theta_{2}, \cdots$.

Definition 7. (Liu [12]) An uncertain random variable is a measurable function $\xi$ from a chance space $(\Gamma, \mathcal{L}, \mathcal{M}) \times(\Omega, \mathcal{A}, \operatorname{Pr})$ to the set of real numbers, i.e., $\xi \in B$ is an event for any Borel set $B$.

Random variables and uncertain variables can be regarded as special cases of uncertain random variables. Let $\eta$ be a random variable, $\tau$ be an uncertain variable. Then $\eta+\tau$ and $\eta \times \tau$ are both uncertain random variables. 
Theorem 4. (Liu [12]) Let $f: R^{n} \rightarrow R$ be a measurable function, and $\xi_{1}, \xi_{2}, \cdots, \xi_{n}$ uncertain random variables on the chance space $(\Gamma, \mathcal{L}, \mathcal{M}) \times(\Omega, \mathcal{A}, \operatorname{Pr})$. Then $\xi=$ $f\left(\xi_{1}, \xi_{2}, \cdots, \xi_{n}\right)$ is an uncertain random variable determined by

$$
\xi(\gamma, \omega)=f\left(\xi_{1}(\gamma, \omega), \xi_{2}(\gamma, \omega), \cdots, \xi_{n}(\gamma, \omega)\right)
$$

for all $(\gamma, \omega) \in \Gamma \times \Omega$.

To calculate the chance measure, Liu [13] presented a definition of chance distribution.

Definition 8. (Liu [13]) Let $\xi$ be an uncertain random variable. Then its chance distribution is defined by

$$
\Phi(x)=\operatorname{Ch}\{\xi \leq x\}
$$

for any $x \in \mathcal{R}$.

The chance distribution of a random variable is just its probability distribution, and the chance distribution of an uncertain variable is just its uncertainty distribution.

Theorem 5. ( $\mathrm{Liu}[13])$ Let $\eta_{1}, \eta_{2}, \cdots, \eta_{m}$ be independent random variables with probability distributions $\Psi_{1}, \Psi_{2}, \cdots, \Psi_{m}$, respectively, and let $\tau_{1}, \tau_{2}, \cdots, \tau_{n}$ be uncertain variables. Then the uncertain random variable $\xi=f\left(\eta_{1}, \eta_{2}, \cdots, \eta_{m}, \tau_{1}, \tau_{2}, \cdots, \tau_{n}\right)$ has a chance distribution

$$
\Phi(x)=\int_{\Re^{m}} F\left(x, y_{1}, \cdots, y_{m}\right) \mathrm{d} \Psi_{1}\left(y_{1}\right) \cdots \mathrm{d} \Psi_{m}\left(y_{m}\right)
$$

where $F\left(x, y_{1}, \cdots, y_{m}\right)$ is the uncertainty distribution of uncertain variable

$$
f\left(\eta_{1}, \eta_{2}, \cdots, \eta_{m}, \tau_{1}, \tau_{2}, \cdots, \tau_{n}\right)
$$

for any real numbers $y_{1}, y_{2}, \cdots, y_{m}$.

Let $\eta$ be a random variable with probability distribution $\Psi$, and let $\tau$ be an uncertain variable with uncertainty distribution $\Upsilon$, by Theorem 5 we proved that the sum $\xi=\eta+\tau$ has a chance distribution

$$
\Phi(x)=\int_{-\infty}^{+\infty} \Upsilon(x-y) \mathrm{d} \Psi(y) .
$$

Let $\eta$ be a positive random variable with probability distribution $\Psi$, and let $\tau$ be a positive uncertain variable with uncertainty distribution $\Upsilon$; by Theorem 5 , we proved that the product $\xi=\eta \tau$ has a chance distribution

$$
\Phi(x)=\int_{0}^{+\infty} \Upsilon(x / y) \mathrm{d} \Psi(y) .
$$

Let $\eta$ be a random variable with probability distribution $\Psi$, and let $\tau$ be an uncertain variable with uncertainty distribution $\Upsilon$; by Theorem 5 , we proved that the minimum $\xi=\eta \wedge \tau$ has a chance distribution

$$
\Phi(x)=\Psi(x)+\Upsilon(x)-\Psi(x) \Upsilon(x) .
$$

Let $\eta$ be a random variable with probability distribution $\Psi$, and let $\tau$ be an uncertain variable with uncertainty distribution $\Upsilon$; by Theorem 5 , we proved that the maximum $\xi=\eta \vee \tau$ has a chance distribution

$$
\Phi(x)=\Psi(x) \Upsilon(x) .
$$


Definition 9. (Liu [13]) Let $\xi$ be an uncertain random variable. Then its expected value is defined by

$$
E[\xi]=\int_{0}^{+\infty} C h\{\xi \geq r\} \mathrm{d} r-\int_{-\infty}^{0} C h\{\xi \leq r\} \mathrm{d} r
$$

provided that at least one of the two integrals is finite.

Let $\Phi$ denote the chance distribution of $\xi$. Liu [13] proved a formula to calculate the expected value of uncertain random variable with chance distribution, that is,

$$
E[\xi]=\int_{0}^{+\infty}(1-\Phi(x)) \mathrm{d} x-\int_{-\infty}^{0} \Phi(x) \mathrm{d} x .
$$

For a random variable $\eta$ and an uncertain variable $\tau$, Liu [13] proved that $E[\eta+\tau]=$ $E[\eta]+E[\tau]$ and $E[\eta \times \tau]=E[\eta] \times E[\tau]$. In fact, we have the following theorem about the expected value of uncertain random variables.

Theorem 6. (Liu [13]) Let $\eta_{1}, \eta_{2}, \cdots, \eta_{m}$ be independent random variables with probability distributions $\Psi_{1}, \Psi_{2}, \cdots, \Psi_{m}$, respectively, and let $\tau_{1}, \tau_{2}, \cdots, \tau_{n}$ be uncertain variables (not necessarily independent), then the uncertain random variable $\xi=$ $f\left(\eta_{1}, \eta_{2}, \cdots, \eta_{m}, \tau_{1}, \tau_{2}, \cdots, \tau_{n}\right)$ has an expected value

$$
E[\xi]=\int_{\Re^{m}} E\left[f\left(y_{1}, y_{2}, \cdots, y_{m}, \tau_{1}, \tau_{2}, \cdots, \tau_{n}\right)\right] \mathrm{d} \Psi_{1}\left(y_{1}\right) \cdots \mathrm{d} \Psi_{m}\left(y_{m}\right)
$$

where $E\left[f\left(y_{1}, y_{2}, \cdots, y_{m}, \tau_{1}, \tau_{2}, \cdots, \tau_{n}\right)\right]$ is the expected value of the uncertain variable $f\left(y_{1}, y_{2}, \cdots, y_{m}, \tau_{1}, \tau_{2}, \cdots, \tau_{n}\right)$ for any real numbers $y_{1}, y_{2}, \cdots, y_{m}$.

Theorem 7. (Liu [13]) Let $\eta_{1}, \eta_{2}, \cdots, \eta_{m}$ be independent uncertain random variables with probability distributions $\Psi_{1}, \Psi_{2}, \cdots, \Psi_{m}$, and let $\tau_{1}, \tau_{2}, \cdots, \tau_{n}$ be independent uncertain random variables with uncertainty distributions $\Upsilon_{1}, \Upsilon_{2}, \cdots, \Upsilon_{n}$, respectively. If $f\left(\eta_{1}, \eta_{2}, \cdots, \eta_{m}, \tau_{1}, \tau_{2}, \cdots, \tau_{n}\right)$ is a strictly increasing or strictly decreasing function with respect to $\tau_{1}, \tau_{2}, \cdots, \tau_{n}$, then the uncertain random variable

$$
\xi=f\left(\eta_{1}, \eta_{2}, \cdots, \eta_{m}, \tau_{1}, \tau_{2}, \cdots, \tau_{n}\right)
$$

has an expected value

$$
E[\xi]=\int_{\Re^{m}} \int_{0}^{1} f\left(y_{1}, \cdots, y_{m}, \Upsilon_{1}^{-1}(\alpha), \cdots, \Upsilon_{n}^{-1}(\alpha)\right) \mathrm{d} \alpha \mathrm{d} \Psi_{1}\left(y_{1}\right) \cdots \mathrm{d} \Psi_{m}\left(y_{m}\right) .
$$

Let $\eta$ be a random variable with probability distribution $\Psi$, and let $\tau$ be an uncertain variable with uncertainty distribution $\Upsilon$. With these, we proved that

$$
E[\eta \vee \tau]=\int_{\Re} \int_{0}^{1}\left(y \vee \Upsilon^{-1}(\alpha)\right) \mathrm{d} \alpha \mathrm{d} \Psi(y)
$$

and

$$
E[\eta \wedge \tau]=\int_{\Re} \int_{0}^{1}\left(y \wedge \Upsilon^{-1}(\alpha)\right) \mathrm{d} \alpha \mathrm{d} \Psi(y) .
$$

\section{Variance of uncertain random variable}

This section will introduce the concept of variance for uncertain random variable as well as a stipulation on the formula to calculate the variance. 
Definition 10. (Liu [13]) Let $\xi$ be an uncertain random variable with a finite expected value $E[\xi]$. Then the variance of $\xi$ is

$$
V[\xi]=E\left[(\xi-E[\xi])^{2}\right] .
$$

Since $(\xi-E[\xi])^{2}$ is a nonnegative uncertain random variable, we also have

$$
V[\xi]=\int_{0}^{+\infty} \operatorname{Ch}\left\{(\xi-E[\xi])^{2} \geq x\right\} \mathrm{d} x .
$$

How to obtain variance from chance distribution? Since the chance measure is a subadditivity measure, the variance of uncertain random variable cannot be derived simply by the chance distribution. In this case, Guo and Wang [14] suggest to a stipulation as follows:

Stipulation 1. (Guo and Wang [14]) Let $\xi$ be an uncertain random variable with a chance distribution $\Phi$. If $\xi$ has a finite expected value $E[\xi]$, then

$$
V[\xi]=\int_{0}^{+\infty}(1-\Phi(E[\xi]+\sqrt{x})+\Phi(E[\xi]-\sqrt{x})) \mathrm{d} x .
$$

Based on this stipulation, we will give some formulas to calculate the variance of an uncertain random variable with the chance distribution and the inverse chance distribution.

Theorem 8. Let $\xi$ be an uncertain random variable with a chance distribution $\Phi$. If $\xi$ has a finite expected value $E[\xi]$, then

$$
V[\xi]=\int_{-\infty}^{+\infty}(x-E[\xi])^{2} \mathrm{~d} \Phi(x) .
$$

Proof: This theorem is based on Stipulation 1 that says the variance of $\xi$ is

$$
V[\xi]=\int_{0}^{+\infty}(1-\Phi(E[\xi]+\sqrt{y})) \mathrm{d} y+\int_{0}^{+\infty} \Phi(E[\xi]-\sqrt{y}) \mathrm{d} y .
$$

Substituting $E[\xi]+\sqrt{y}$ with $x$ and $y$ with $(x-E[\xi])^{2}$, the change of variables and integration by parts produced, we have

$$
\int_{0}^{+\infty}(1-\Phi(E[\xi]+\sqrt{y})) \mathrm{d} y=\int_{E[\xi]}^{+\infty}(1-\Phi(x)) \mathrm{d}(x-E[\xi])^{2}=\int_{E[\xi]}^{+\infty}(x-E[\xi])^{2} \mathrm{~d} \Phi(x) .
$$

Similarly, substituting $e-\sqrt{y}$ with $x$ and $y$ with $(x-e)^{2}$, we obtain

$$
\int_{0}^{+\infty} \Phi(E[\xi]-\sqrt{y}) \mathrm{d} y=\int_{E[\xi]}^{-\infty} \Phi(x) \mathrm{d}(x-E[\xi])^{2}=\int_{-\infty}^{E[\xi]}(x-E[\xi])^{2} \mathrm{~d} \Phi(x) .
$$

It follows that the variance is

$$
V[\xi]=\int_{E[\xi]}^{+\infty}(x-E[\xi])^{2} \mathrm{~d} \Phi(x)+\int_{-\infty}^{E[\xi]}(x-E[\xi])^{2} \mathrm{~d} \Phi(x)=\int_{-\infty}^{+\infty}(x-E[\xi])^{2} \mathrm{~d} \Phi(x) .
$$

The theorem is verified. 
Theorem 9. Let $\xi$ be an uncertain random variable with a regular chance distribution $\Phi$. If $\xi$ has a finite expected value $E[\xi]$, then

$$
V[\xi]=\int_{0}^{1}\left(\Phi^{-1}(r)-E[\xi]\right)^{2} \mathrm{~d} r .
$$

Proof: Substituting $\Phi(x)$ with $r$ and $x$ with $\Phi^{-1}(r)$, it follows from the change of variables of integration and Theorem 8 that the variance is

$$
V[\xi]=\int_{-\infty}^{+\infty}(x-E[\xi])^{2} \mathrm{~d} \Phi(x)=\int_{0}^{1}\left(\Phi^{-1}(r)-E[\xi]\right)^{2} \mathrm{~d} r .
$$

The theorem is verified.

Example 1. Let $\eta$ be a random variable with probability distribution $\Psi$, and let $\tau$ be an uncertain variable with uncertainty distribution $\Upsilon$. Then by Theorem 8 , the sum $\xi=\eta+\tau$ has the variance

$$
V[\xi]=\int_{-\infty}^{+\infty}(x-E[\xi])^{2} \mathrm{~d} \Phi(x)
$$

where $\Phi(x)=\int_{-\infty}^{+\infty} \Upsilon(x-y) \mathrm{d} \Psi(y)$ and $E[\xi]=E[\eta]+E[\tau]$. By Theorem 9 , the sum $\xi=$ $\eta+\tau$ has the variance

$$
V[\xi]=\int_{0}^{1}\left(\Phi^{-1}(r)-(E[\eta]+E[\tau])\right)^{2} \mathrm{~d} r .
$$

Example 2. Let $\eta$ be a random variable with probability distribution $\Psi$, and let $\tau$ be an uncertain variable with uncertainty distribution $\Upsilon$. Then by Theorem 8 , the product $\xi=\eta \tau$ has the variance

$$
V[\xi]=\int_{-\infty}^{+\infty}(x-E[\xi])^{2} \mathrm{~d} \Phi(x)
$$

where $\Phi(x)=\int_{-\infty}^{+\infty} \Upsilon(x / y) \mathrm{d} \Psi(y)$ and $E[\xi]=E[\eta] E[\tau]$. By Theorem 9 , the product $\xi=$ $\eta \tau$ has the variance

$$
V[\xi]=\int_{0}^{1}\left(\Phi^{-1}(r)-E[\eta] E[\tau]\right)^{2} \mathrm{~d} r .
$$

Example 3. Let $\eta$ be a random variable with probability distribution $\Psi$, and let $\tau$ be an uncertain variable with uncertainty distribution $\Upsilon$. Then by Theorem 8 , the minimum $\xi=\eta \wedge \tau$ has the variance

$$
V[\xi]=\int_{-\infty}^{+\infty}(x-E[\xi])^{2} \mathrm{~d} \Phi(x)
$$

where $\Phi(x)=\Psi(x)+\Upsilon(x)-\Psi(x) \Upsilon(x)$ and $E[\xi]=E[\eta \wedge \tau]=\int_{\Re} \int_{0}^{1}\left(y \wedge \Upsilon^{-1}(\alpha)\right) \mathrm{d} \alpha \mathrm{d} \Psi(y)$. By Theorem 9, the minimum $\xi=\eta \wedge \tau$ has the variance

$$
V[\xi]=\int_{0}^{1}\left(\Phi^{-1}(r)-E[\eta \wedge \tau]\right)^{2} \mathrm{~d} r .
$$


Example 4. Let $\eta$ be a random variable with probability distribution $\Psi$, and let $\tau$ be an uncertain variable with uncertainty distribution $\Upsilon$. Then by Theorem 8 , the maximum $\xi=\eta \vee \tau$ has the variance

$$
V[\xi]=\int_{-\infty}^{+\infty}(x-E[\xi])^{2} \mathrm{~d} \Phi(x)
$$

where $\Phi(x)=\Psi(x) \Upsilon(x)$ and $E[\xi]=E[\eta \vee \tau]=\int_{\Re} \int_{0}^{1}\left(y \vee \Upsilon^{-1}(\alpha)\right) \mathrm{d} \alpha \mathrm{d} \Psi(y)$. By Theorem 9, the minimum $\xi=\eta \vee \tau$ has the variance

$$
V[\xi]=\int_{0}^{1}\left(\Phi^{-1}(r)-E[\eta \vee \tau]\right)^{2} \mathrm{~d} r .
$$

\section{Conclusions}

In this paper, we mainly studied the variance of an uncertain random variable. Some formulas were derived to calculate the variance of an uncertain random variable using chance distribution and the inverse chance distribution. At the same time, several practical examples are also provided to calculate the variance of uncertain random variables with chance distribution and inverse chance distribution.

\footnotetext{
Acknowledgements

This work was supported by National Natural Science Foundation of China Grants No.61273044 and No.91224008.

\section{Author details}

${ }^{1}$ Department of Mathematical Sciences, Tsinghua University, Beijing 100084, China. ${ }^{2}$ School of Management, University of Chinese Academy of Sciences, Beijing 100190, China.
}

Received: 4 March 2014 Accepted: 16 April 2014

Published: 7 May 2014

\section{References}

1. Kolmogorov, AN: Grundbegriffe der Wahrscheinlichkeitsrechnung. Julius Springer, Berlin (1933)

2. Kahneman, D, Tversky, A: Prospect theory: an analysis of decision under risk. Econometrica. 47(2), 263 - 292 (1979)

3. Liu, B: Uncertainty Theory: A Branch of Mathematics for Modeling Human Uncertainty. Springer-Verlag, Berlin (2010)

4. Liu, B: Why is there a need for uncertainty theory? J. Uncertain Syst. 6(1), 3-10 (2012)

5. Liu, B: Uncertainty Theory. 2nd ed. Springer-Verlag, Berlin (2007)

6. Liu, B: Some research problems in uncertainty theory. J. Uncertain Syst. 3(1), 3-10 (2009)

7. Gao, X: Some properties of continuous uncertain measure. Int. J. Uncertainty Fuzziness Knowledge-Based Syst. 17(3), 419-426 (2009)

8. Peng, ZX, Iwamura, K: A sufficient and necessary condition of uncertainty distribution. J. Interdiscip. Math. 13(3), 277-285 (2010)

9. Liu, YH, Ha, MH: Expected value of function of uncertain variables. J. Uncertain Syst. 4(3), 181_186 (2010)

10. Yao, K: A formula to calculate the variance of uncertain variable. http://orsc.edu.cn/online/130831.pdf.

11. Sheng, YH, Samarjit, K: Some results of moments of uncertain variable through inverse uncertainty distribution. Fuzzy Optimization Decis. Making. (2014 in press)

12. Liu, YH: Uncertain random variables: a mixture of uncertainty and randomness. Soft Comput. 17(4), 625-634 (2013)

13. Liu, YH: Uncertain random programming with applications. Fuzzy Optimization Decis. Making. 12(2), 153-169 (2013)

14. Guo, HY, Wang, XS: Variance of uncertain random variables. J. Uncertainty Anal. Appl. (2014 in press)

15. Yao, K, Gao, JW: Law of large numbers for uncertain random variables. http://orsc.edu.cn-/online/120401.pdf.

16. Gao, JW, Yao, K: Some concepts and theorems of uncertain random process. Int. J. Intell. Syst. (2014 in press)

17. Hou, YC: Subadditivity of chance measure. http://orsc.edu.cn/online/130602.pdf.

doi:10.1186/2195-5468-2-12

Cite this article as: Sheng and Yao: Some formulas of variance of uncertain random variable. Journal of Uncertainty Analysis and Applications 2014 2:12. 